\title{
Las especies del género Gyropus Nitzsch, 1818 (Phthiraptera: Gyropidae) parásitas de Octodontidae (Mammalia: Rodentia)
}

\author{
The species of the genus Gyropus Nitzch, 1818 (Phthiraptera: Gyropidae) parasitic on \\ the Octodontidae (Mammalia: Rodentia)
}

\begin{abstract}
DOLORES DEL CARMEN CASTRO ${ }^{1} \&$ ARMANDO CICCHINO $^{2}$
${ }^{1}$ Facultad de Ciencias Naturales y Museo de La Plata, Paseo del Bosque s/n, 1900 La Plata, Buenos

Aires, Argentina; e-mail: castrodreon@Impsat1.com.ar

${ }^{2}$ Departamento Científico de Entomología, Museo de La Plata, Paseo del Bosque s/n, 1900 La Plata, Buenos Aires, Argentina; e-mail: cicchino@museo.fcnym.unlp.edu.ar
\end{abstract}

\begin{abstract}
RESUMEN
Este trabajo tiene por finalidad otorgar status específico pleno a la subespecie Gyropus parvus elongatus Castro, Cicchino \& Torres-Mura, 1987, describir la nueva especie Gyropus distinctus a partir de materiales recolectados sobre Octodon degus (Molina, 1782) y Octodon lunatus Osgood, 1943 procedentes del centro de Chile y, finalmente, ofrecer un análisis de las posibles razones de la distribución geográfica y del rango de hospedadores conocidos para estas tres especies.
\end{abstract}

Palabras clave: Phthiraptera, Gyropus, Octodontidae.

\section{ABSTRACT}

The purpose of this paper is to give full specific status to the subspecies Gyropus parvus elongatus Castro, Cicchino \& Torres-Mura, 1987, to describe the new species Gyropus distinctus collected in Octodon degus and O. lunatus from central Chile, along with an analysis of the possible causes than could explain their geographic and host range distributions known to date for these three species.

Key words: Phthiraptera, Gyropus, Octodontidae.

\section{INTRODUCCIÓN}

Hasta el presente, una especie que incluye dos subespecies ha sido formalmente descrita como parásita de esta familia de roedores neotropicales. El primer aporte fue el pionero trabajo de Ewing (1924) quien describe Monogyropus parvus, especie parásita de Ctenomys colburni (J.A. Allen) de Huanuluán, provincia de Río Negro, Argentina. Más tarde, esta especie fue reestudiada por Werneck (1936, 1948), quien la incluyó en el género Gyropus Nitzsch, 1818. Poco tiempo más tarde, este mismo autor (Werneck 1951) adjudicó transitoriamente a esta especie especímenes recolectados sobre Octodon degus (Molina, 1782) procedentes de Santiago, Chile, señalando sus diferencias con materiales recolectados en Ctenomys spp. Más recientemente Castro et al. (1987) dividieron a esta especie en dos subespecies, G. p. parvus, que parasita exclusivamente a especies del género Ctenomys (Ctenomyinae) y G. p. elongatus Castro, Cicchino \& Torres-Mura, que parasita a Aconaemys fuscus
(Octodontinae). Cicchino \& Castro (1994) reestudiaron la primera de ellas, analizando su distribución en 10 especies de Ctenomys y su sinoxenismo con especies del género Phtheiropoios Eichler, 1939. Finalmente, Cicchino et al. (en prensa) hicieron una revisión del status de todas las poblaciones hasta ese momento conocidas que parasitan Ctenomys, incluyendo a $G$. parvus.

En este estudio es nuestro propósito darle a ambas status específico pleno, ampliar sus descripciones para su más adecuado reconocimiento, describir una nueva especie parásita de Octodon degus (Molina, 1782) y O. lunatus Osgood, 1943, y ofrecer un análisis de la distribución en las especies de hospedadores de las tres especies.

\section{MATERIALES Y MÉTODOS}

Los ejemplares estudiados fueron obtenidos directamente sobre los hospederos mencionados en cada caso, y montados en preparaciones micros- 
cópicas permanentes, siguiendo la metodología propuesta por Castro \& Cicchino (1978).

Las dimensiones corporales se expresan en milímetros, y constan del largo de la cabeza (HL), ancho máximo de la misma (HW), largo del abdomen (AL), ancho máximo del mismo (AW), y largo total del cuerpo (TL). Para cada una de ellas se señala el rango y, entre paréntesis, el promedio y desviación estándar de cada muestra.

En la notación de la quetotaxia abdominal se ha tenido en cuenta que en la mayoría de los segmentos hay dos hileras de setas, de manera que para poder diferenciarlos en cada uno de los segmentos, el conteo correspondiente a la hilera anterior se muestra entre paréntesis, y el de la posterior sin ellos. Para ambos guarismos, se señalan sus respectivos rangos, observados en toda la serie estudiada.

Las ilustraciones fueron realizadas por los autores con cámara clara a distintos aumentos. Las escalas utilizadas en las figuras, siempre están expresadas en micrómetros $(\mu \mathrm{m})$.

La totalidad del material estudiado se encuentra depositado en las colecciones del Museo de La Plata, La Plata, provincia de Buenos Aires, Argentina (MLP), bajo el número: 2652.

\section{RESULTADOS}

Gyropus parvus (Ewing, 1924).

Monogyropus parvus Ewing (1924) Proceedings of the United States National Museum 63: 11-12; Fig. 5.

Gyropus parvus, Werneck, 1936, Mem. Inst. Oswaldo Cruz, 31: 454-456, Figs. 85-86, Werneck, 1948, Os malofagos de mamíferos I: 70-71; Fig. 68-69.

Gyropus parvus parvus (Ewing, 1924); Castro, Cicchino \& Torres-Mura (1987) Anales del Museo de Historia Natural de Valparaíso (Chile) 18: 41-45; Cicchinio \& Castro (1994) Iheringia, Serie Zoología 77: 4; Fig. 1-3, 14-24, 31-35.

El estudio de nuevos materiales pertenecientes a distintas poblaciones locales referibles al menos a 10 especies de Ctenomys (Cicchino et al. en prensa) reveló una marcada constancia en los caracteres quetotácticos (Tabla 1) y morfométricos (Tabla 2), ya anticipados por Castro et al. (1987) y Cicchino \& Castro (1994), por lo que consideramos a todas ellas como pertenecientes al mismo taxón específico.

TABLA 1

Quetotaxia tergal (A) y esternal (B) del abdomen de las tres especies de Gyropus tratadas en este trabajo, y correspondientes a machos y hembras respectivamente

Tergal (A) and sternal (B) chaetotaxy of the abdomen of the three species of Gyropus dealt with in this paper, males and females, respectively

(A)

\begin{tabular}{llllllll}
\hline $\begin{array}{l}\text { Machos } \\
\text { tergal }\end{array}$ & G. parvus & G. elongatus & G. distinctus & $\begin{array}{l}\text { Hembras } \\
\text { tergal }\end{array}$ & G. parvus & G. elongatus & G. distinctus \\
\hline Tergito I & $5-8 / 10-12$ & $10-13 / 13-15$ & $13-16 / 16-19$ & Tergito I & $7-10 / 12-14$ & $13-14 / 16-17$ & $12-14 / 18-19$ \\
Tergito II & $7-11 / 17-18$ & $13-15 / 22-24$ & $13-16 / 25-28$ & Tergito II & $8-10 / 17-24$ & $17-18 / 28-29$ & $15-22 / 34-42$ \\
Tergito III & $7-10 / 17-23$ & $16-18 / 25-26$ & $14-16 / 30-32$ & Tergito III & $7-12 / 23-32$ & $20-24 / 32-35$ & $19-24 / 38-49$ \\
Tergito IV & $9-12 / 19-22$ & $18 / 23-28$ & $14-18 / 28-31$ & Tergito IV & $10-12 / 24-28$ & $20-22 / 32-33$ & $21-22 / 40-50$ \\
Tergito V & $9-12 / 15-21$ & $13-19 / 22-27$ & $15-16 / 26-31$ & Tergito V & $10-13 / 23-25$ & $20-25 / 33-37$ & $21-27 / 40-47$ \\
Tergito VI & $8-12 / 12-14$ & $15-17 / 17-19$ & $15-17 / 21-22$ & Tergito VI & $10-13 / 15-19$ & $19-21 / 25-30$ & $20-28 / 26-40$ \\
Tergito VII & $5-7 / 6-8$ & $8-10 / 8-10$ & $10-13 / 10-12$ & Tergito VII & $8-10 / 7-10$ & $15-16 / 12-13$ & $11-17 / 11-18$ \\
Tergito VIII & $2-4 / 0-2$ & $2-3 / 0-2$ & $2-3 / 2$ & Tergito VIII & $2 / 2$ & $3 / 2$ & $2-3 / 1-2$
\end{tabular}

(B)

\begin{tabular}{llllllll}
\hline $\begin{array}{l}\text { Machos } \\
\text { esternal }\end{array}$ & G. parvus & G. elongatus & G.distinctus & $\begin{array}{l}\text { Hembras } \\
\text { esternal }\end{array}$ & G. parvus & G. elongatus & G. distinctus \\
\hline Esternito I & $0-3 / 6$ & $4-5 / 67$ & $3-7 / 6-11$ & Esternito I & $2-3 / 6-7$ & $3-4 / 7-8$ & $4-6 / 7-9$ \\
Esternito II & $4-7 / 18-20$ & $12-14 / 20-22$ & $9-11 / 21-24$ & Esternito II & $5-7 / 18-20$ & $11-14 / 20-22$ & $11-15 / 28-30$ \\
Esternito III & $10-15 / 19-25$ & $13-17 / 20-24$ & $11-14 / 26-29$ & Esternito III & $8-13 / 23-25$ & $14-15 / 25-27$ & $14-15 / 25-31$ \\
Esternito IV & $11-14 / 19-25$ & $12-17 / 20-22$ & $12-15 / 22-28$ & Esternito IV & $12-15 / 23-29$ & $16-17 / 22-24$ & $15-16 / 28-32$ \\
Esternito V & $9-14 / 17-20$ & $12-14 / 17-26$ & $11-17 / 17-20$ & Esternito V & $11-14 / 19-22$ & $16-17 / 22-23$ & $13-15 / 26-29$ \\
Esternito VI & $8-12 / 12-16$ & $12-14 / 13-17$ & $10-12 / 13-18$ & Esternito VI & $10-14 / 14-18$ & $14-17 / 17-20$ & $12-13 / 18-20$ \\
Esternito VII & $6-9 / 7-8$ & $5-11 / 5-10$ & $4-7 / 8-9$ & Esternito VII & $9-12 / 8-11$ & $10-11 / 8-16$ & $11-12 / 8-11$ \\
Esternito VIII & $1-2 / 2$ & $2 / 2$ & $2 / 0$ & & & & \\
\hline
\end{tabular}


Gyropus elongatus Castro, Cicchino \& TorresMura, 1987, status novo.

Gyropus parvus elongatus Castro, Cicchino \& Torres-Mura (1987) Anales del Museo de Historia Natural de Valparaíso (Chile) 18: 41-45.

Este taxón fue originalmente descrito como una subespecie de G. parvus. Una revalorización de la constancia de sus características morfológicas en relación con las exhibidas por G. parvus y con la que aquí se describe a continuación, sumada a las consideraciones que señalamos más adelante en la discusión acerca de las relaciones de éstas con sus respectivos hospederos, justifican el nuevo status taxonómico que proponemos.

Gyropus distinctus n. sp., Fig. 1A, 1B, 1C y $2 \mathrm{~A}$.

Diagnosis: especie morfológicamente muy similar a $G$. elongatus difiriendo en ambos sexos por presentar mayor tamaño, en el macho por las características del pseudopene y la armadura del saco genital y la presencia de un mayor numero de setas en los tergitos II-IV, y en las hembras por un mayor número de setas en los tergitos y esternitos II-V.

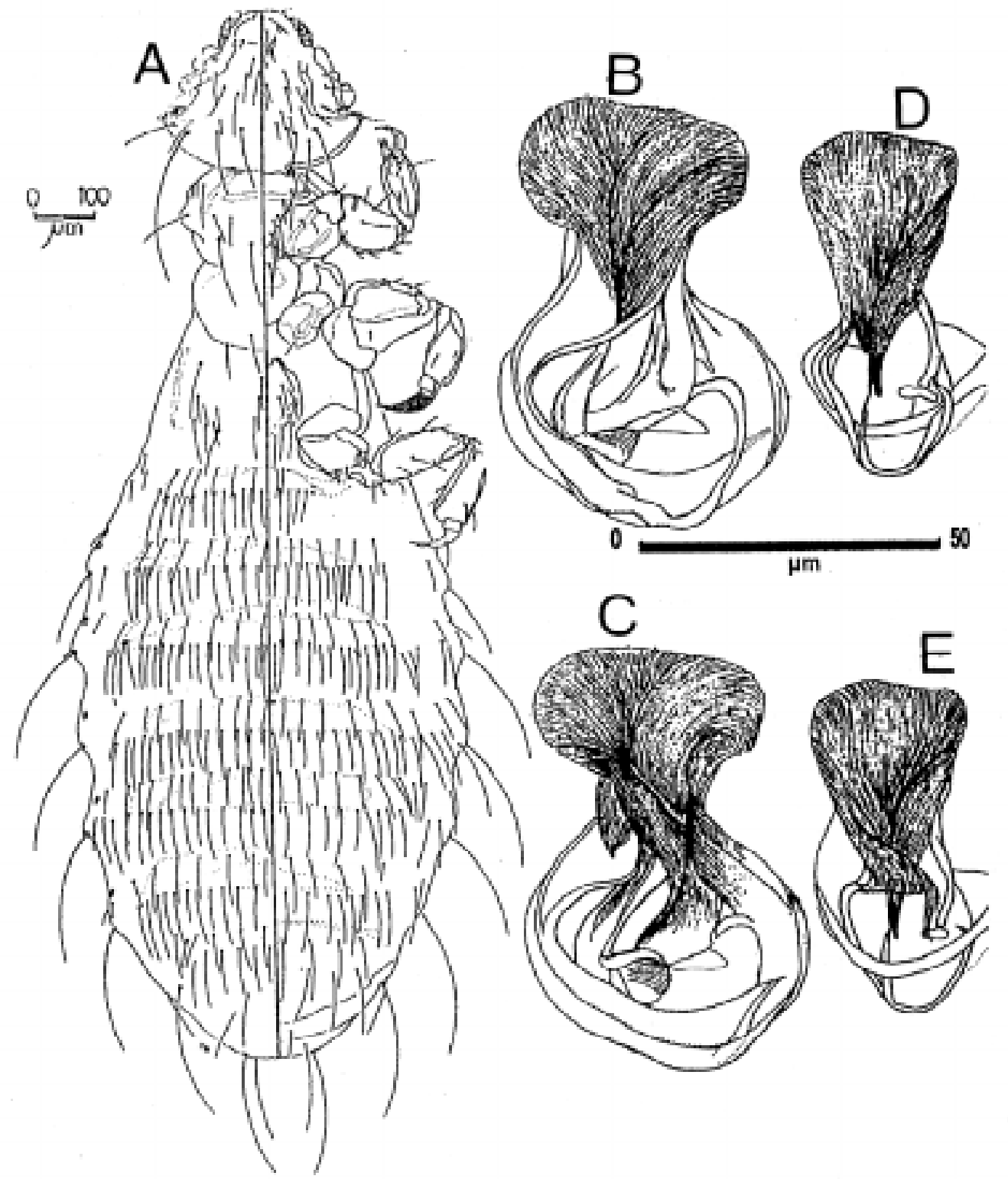

Fig. 1: (A, B, C) Gyropus distinctus n. sp.: (A) macho, dorsal y ventral; (B, C) armadura del saco genital; (D, E) armadura del saco genital de Gyropus elongatus.

(A, B, C) Gyropus distinctus n. sp.: (A) male, dorsal and ventral views; (B, C) spiculation of the genital sac; (D, E) spiculation of the genital sac of Gyropus elongatus. 
Etimología: alude a sus diferencias respecto de G. elongatus

\section{Descripción}

Macho: aspecto general como en la Fig. 1. Silueta corporal muy alargada reminiscente a la de $G$. elongatus, diferenciándose por su mayor longitud corporal y mayor tamaño absoluto de la cabeza (Tabla 2). Además muestra una acusada tendencia a un incremento del número de setas en los tergitos II-IV (Tabla 1). Genitales externos: pseudopene en forma de manubrio caudado, (Fig. 2A) y armadura del saco genital distintiva respecto de la de G. elongatus, como se muestra en las Fig. 1B, 1C, y 1D y 1E, respectivamente.

Hembra: aspecto general semejante al macho. Silueta corporal comparable con la de $G$. elongatus, diferenciándose por su mayor talla corporal y mayor tamaño absoluto de la cabeza (Tabla 2), además muestra un mayor número de setas en los tergitos y esternitos abdominales IIV (Tabla 1).

Hospedero tipo: Octodon degus (Molina, 1782). Otro hospedero: Octodon lunatus Osgood, 1943.

Material examinado: holotipo macho, 12 machos y 20 hembras paratipos,(MLP 2652) Reserva Nacional Lago Peñuelas, Valparaíso, Chile, 26-I1988, J.C. Torres-Mura, todos sobre su hospedero tipo. Otros materiales, no considerados tipos: tres machos y dos hembras, Reserva Nacional
Lago Peñuelas, Valparaíso, Chile, 2-VII-1987, J.C. Torres-Mura, sobre $O$. lunatus.

Observaciones: esta especie ha sido atribuida a Gyropus parvus por Werneck (1951), quien señaló también las diferencias con los ejemplares procedentes de Ctenomys referidas a su particular morfo, y las que observó en el aparato copulador masculino.

\section{DISCUSIÓN Y CONCLUSIONES}

Gyropus parvus está ampliamente distribuida en más de 10 especies de Ctenomys (Cicchino \& Castro 1994, Cicchino et al. en prensa), y presenta una gran uniformidad morfológica y métrica entre las distintas "poblaciones" que parasitan distintas especies de este género. Todas ellas presentan un morfo corporal acortado y tergitos y esternitos guarnecidos por dos hileras de setas cuyo número oscila, en los tergitos II-V entre 523 para los machos y 7-32 para las hembras (Tabla 1). Gyropus elongatus y G. distinctus sp. nov. presentan un morfo corporal alargado en ambos sexos y una manifiesta tendencia al incremento del número de setas, sobre todo en estos segmentos, $10-18$ y $13-32$ para los machos y $13-37$ y 12 50 para las hembras, respectivamente.

Este alargamiento de la silueta corporal por parte de estas dos últimas especies posiblemente sea el reflejo de un nicho diferente al ocupado por G. parvus (Castro et al. 1987). Esta especializa-

TABLA 2

Medidas corporales (en $\mathrm{mm}$ ) de las tres especies de Gyropus tratadas en este trabajo, y correspondientes a (A) machos y (B) hembras, respectivamente

Body measurements (in mm) of the three species of Gyropus dealt with in this paper, (A) males and (B) females, respectively

(A)

\begin{tabular}{lccccccccc}
\hline Machos & \multicolumn{3}{c}{ Gyropus parvus } & \multicolumn{3}{c}{ Gyropus elongatus } & \multicolumn{3}{c}{ Gyropus distinctus } \\
& Rango & Media & DE & Rango & Media & DE & Rango & Media & DE \\
\hline Largo máximo de la cabeza & $0,231-0,242$ & 0,235 & 0,005 & $0,231-0,247$ & 0,238 & 0,007 & $0,263-0,267$ & 0,265 & 0,002 \\
Ancho máximo de la cabeza & $0,265-0,277$ & 0,275 & 0,005 & $0,282-0,300$ & 0,289 & 0,006 & $0,287-0,313$ & 0,305 & 0,013 \\
Ancho máximo del abdomen & $0,674-0,709$ & 0,694 & 0,013 & $0,508-0,542$ & 0,519 & 0,012 & $0,520-0,573$ & 0,552 & 0,020 \\
Largo total del cuerpo & $1,280-1,292$ & 1,290 & 0,005 & $1,505-1,569$ & 1,531 & 0,022 & $1,753-1,813$ & 1,779 & 0,026
\end{tabular}

(B)

\begin{tabular}{lccccccccccc}
\hline Hembras & \multicolumn{4}{c}{ Gyropus parvus } & \multicolumn{3}{c}{ Gyropus elongatus } & \multicolumn{3}{c}{ Gyropus distinctus } \\
& Rango & Media & DE & Rango & Media & DE & Rango & Media & DE & \\
\hline Largo máximo de la cabeza & $0,247-0,254$ & 0,250 & 0,004 & $0,254-0,266$ & 0,266 & 0,006 & $0,289-0,295$ & 0,292 & 00,003 \\
Ancho máximo de la cabeza & $0,277-0,300$ & 0,289 & 0,010 & $0,300-0,323$ & 0,308 & 0,009 & $0,347-0,360$ & 0,348 & 0,010 \\
Ancho máximo del abdomen & $0,535-0,565$ & 0,550 & 0,013 & $0,589-0,692$ & 0,610 & 0,040 & $0,689-0,800$ & 0,738 & 0,040 \\
Largo total del cuerpo & $1,442-1,535$ & 1,535 & 0,049 & $1,812-1,978$ & 1,874 & 0,061 & $2,316-2,443$ & 2,345 & 0,068 \\
\hline
\end{tabular}


ción corporal corre paralela a un soporte pilífero de longitud mayor al observado en Ctenomys como es el caso de Aconaemys y Octodon (Redford \& Eisenberg 1989). Por esta razón este morfo pudo ser consecuencia de una de dos alternativas. La primera presupondría un origen filogenético común de las estirpes que condujeron a Aconaemys y Octodon por una parte y Ctenomys por la otra.

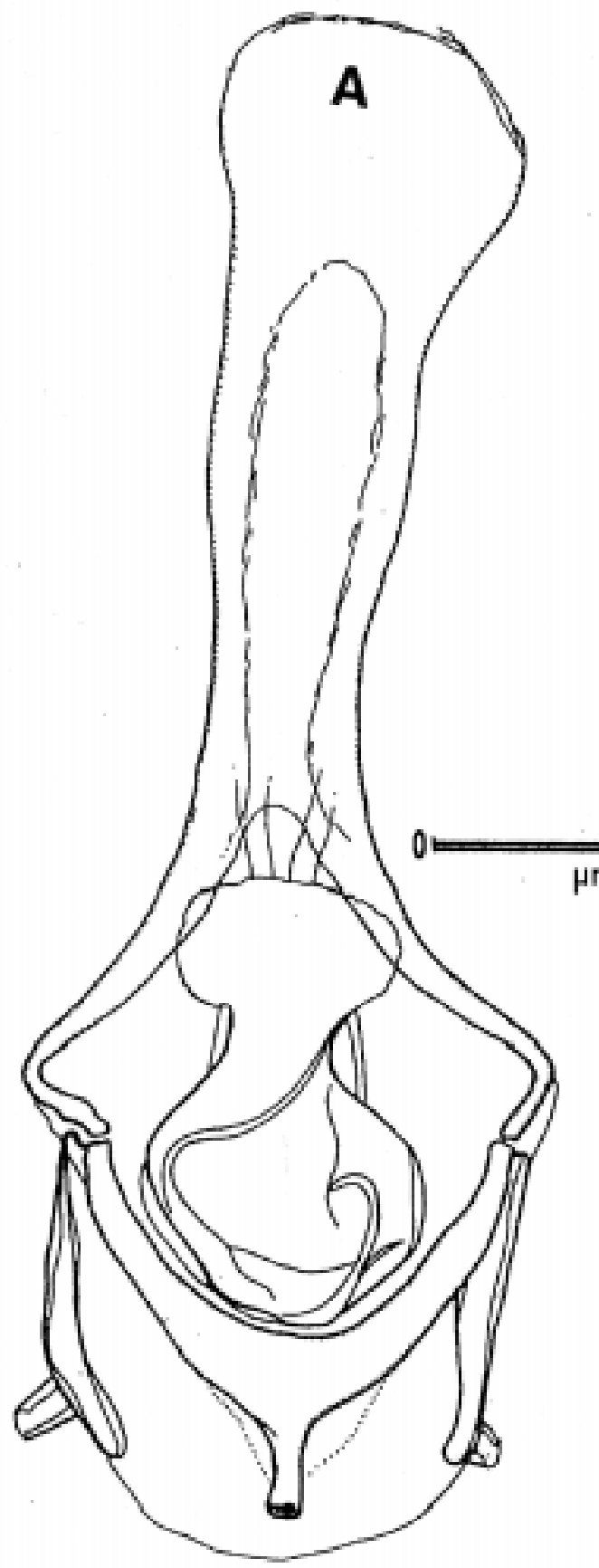

La segunda se originaría en una colonización secundaria, contemporánea o extemporánea de miembros de las estirpes que finalmente condujeron a Aconaemys y Octodon, a partir de un "stock ctenomino". Entonces, G. elongatus y G. distinctus no serían especies hermanas sino especies derivadas de un tronco común que comparten un morfo corporal que solamente indicaría similitud de ni- 
chos, teniendo en cuenta además que los hospederos hasta hoy conocidos para una y otra especie son también alopátricos. Los dos conocidos para G. distinctus, Octodon degus y O. lunatus, son parcialmente simpátricos en la región central de Chile (Redford \& Eisenberg 1992), pero no tienen los mismos hábitos. O. lunatus aparentemente es una especie menos fosorial y que habita las áreas con matorrales más húmedas que $O$. degus, por lo que el factor hídrico sería un factor importante en la determinación de la distribución de ambas especies (Contreras et al. 1987). No obstante, estas diferencias ambientales y de comportamiento parecen no haber influido en la diferenciación de ambas poblaciones de Gyropus, las que son morfológicamente indistinguibles, y por ello atribuidas a la misma especie taxonómica. En lo que respecta al único hospedero conocido para $G$. elongatus, Aconaemys fuscus, es una especie que exhibe una gran tolerancia ambiental por lo que se la halla desde estipales y festucales subxéricos hasta bosques de Nothofagus y Araucaria del centro de Chile y el centrooeste de la Argentina (Redford \& Eisenberg 1992), y es de hábitos subfosoriales (Contreras et al. 1987) y, como ya se ha dicho, parece ser alopátrica respecto de ambas especies de Octodon. Esta alopatría habría contribuido también en el aislamiento genético de esta especie de Gyropus en relación con la anterior, ya que solamente cohabita con Akodon longipilis y Notiomys valdivianus (Redford \& Eisenberg 1992), ambos roedores sigmodontinos parasitados únicamente por especies de Hoplopleura Enderlein, 1904 (Anoplura: Hoplopleuridae).

\section{LITERATURA CITADA}

CASTRO D DEL C \& AC CICCHINO (1978) Contribución al conocimiento de los Malófagos argentinos III. Sobre algunos Menoponidae de la avifauna bonaerense: Menacanthus eurysternus (Burmeister) y Menacanthus pici (Denny) (Insecta-Mallophaga). Revista de la Sociedad Entomológica Argentina 37: 77-83.

CASTRO D DEL C, AC CICCHINO \& JC TORRESMURA (1987) Estudio descriptivo y comparativo de Gyropus parvus (Mallophaga), parásito de roedores octodontoideos. Anales del Museo de Historia Natural de Valparaíso (Chile) 18: 41-45.

CICCHINO AC \& D DEL C CASTRO (1994) On Gyropus parvus parvus (Ewing, 1924) and Phtheiropoios rionegrensis sp. n. (Phthiraptera, Amblycera, Gyropidae) parasitic on Ctenomys haigi Thomas, 1919 (Mammalia, Rodentia, Ctenomyidae). Iheringia, Série Zoologia 77: 3-14.

CICCHINO A, D DEL C CASTRO \& JL BALDO (en prensa) Elenco de los Phthiraptera (Hexapoda) hallados en distintas poblaciones locales de Ctenomys (Rodentia) de Argentina, Uruguay, Paraguay y Brasil. Arquivos de Zoología, Museu de Zoología da Universidade de Sâo Paulo.

CONTRERAS LC, JC TORRES-MURA \& JL YÁÑEZ (1987) Biogeography of octodontid rodents: an ecoevolutionary hypothesis. Fieldiana Zoology 39: 401411.

EWING HE (1924) On the taxonomy, biology, and distribution of the biting lice of the family Gyropidae. Proceedings of the United States National Museum 63: $1-42$.

REDFORD KH \& JF EISENBERG (1992) Mammals of the Neotropics: the southern cone. University of Chicago Press, Chicago, Illinois. $430 \mathrm{pp}$.

WERNECK FL (1936) Contribuiçâo ao conhecimento dos mallophagos encontrados nos mamíferos sul-americanos. Memorias do Instituto Oswaldo Cruz 31: 391 589.

WERNECK FL (1948) Os Mallophagos de mammiferos. Parte I. Amblycera e Ischnocera (Philopteridae e parte de Trichodectidae). Revista Brasileira de Biología, Río de Janeiro, 243 pp.

WERNECK FL (1951) Notas sôbre malófagos (Gyropidae). Revista Brasileira de Biologia 11: 303-313.

Editor Asociado: M. George-Nascimento

Recibido el 4 de mayo de 2000; aceptado el 21 de diciembre de 2001 